\title{
Five-year results of the pilot trial of a sutureless valve
}

\author{
Bart Meuris, MD, PhD, ${ }^{a}$ Willem J. Flameng, MD, PhD, ${ }^{a}$ François Laborde, MD, ${ }^{\mathrm{b}}$ \\ Thierry A. Folliguet, MD, ${ }^{b}$ Axel Haverich, $\mathrm{MD},{ }^{\mathrm{c}}$ and Malakh Shrestha, MD, $\mathrm{PhD}$
}

\section{ABSTRACT}

Objective: A prospective trial was designed to evaluate the feasibility of the Perceval sutureless aortic valve. We report the 5-year clinical and hemodynamic outcome.

Methods: A total of 30 patients (mean age: $80.4 \pm 3.8$ years; mean logistic European System for Cardiac Operative Risk Evaluation [euroSCORE]: $13.2 \pm 7.3$ ) received the valve in 3 European centers, between April 2007 and February 2008. Cumulative follow-up was 92.67 patient-years, with a median of 4.2 years. Patients with a small annulus were selected because only sizes 21 and $23 \mathrm{~mm}$ (covering annuli diameters from 19 to $23 \mathrm{~mm}$ ) were available at this early stage of the trial. In $37 \%$ of the patients, a $21-\mathrm{mm}$ valve was used; $63 \%$ received a 23 -mm valve; 14 patients had concomitant coronary artery bypass grafting. Clinical and hemodynamic follow-up evaluation were performed annually, including echocardiography.

Results: Procedural success was $100 \%$. Cardiopulmonary bypass time and crossclamp time in isolated aortic valve replacement were $46.4 \pm 6.7$ minutes and 29.3 \pm 8.0 minutes, respectively. One patient died during the hospital stay. Postoperative complications included 1 patient with mediastinal bleeding, and 1 with atrioventricular block that led to pacemaker implantation. No stroke occurred in either the early or late period. At the last available follow-up, 22 patients were alive. The mean gradient was $9.3 \mathrm{~mm} \mathrm{Hg}$, with an effective orifice area of $1.7 \mathrm{~cm}^{2}$ at 5 years. No dislodgement, structural valve deterioration, hemolysis, or valve thrombosis was reported.

Conclusions: This study reports the first and longest experience with a truly sutureless valve, evaluating implantation feasibility and valve safety. Results from up to 5 years of follow up confirmed the performance and safety of this device, even in a medium- to high-risk patient population with a small aortic annulus. (J Thorac Cardiovasc Surg 2015;150:84-8)

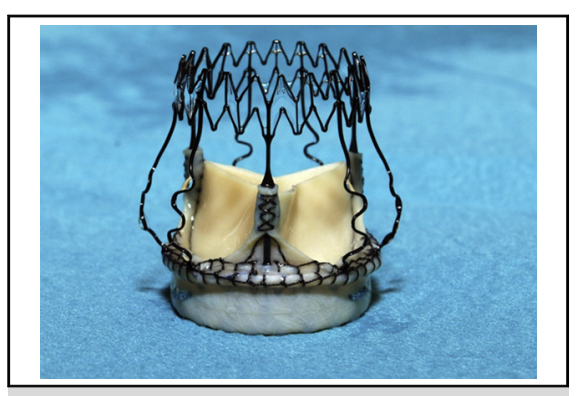

The Perceval bioprosthesis is currently, worldwide, the most frequently used sutureless valve.

\section{Central Message}

Five-year outcomes of a sutureless aortic valve in 30 elderly patients showed survival at $71.3 \%$, and a mean gradient of $9.3 \mathrm{~mm} \mathrm{Hg}$. Effective orifice area was $1.7 \mathrm{~cm}^{2}$, without dislodgement, structural valve deterioration, hemolysis, or valve thrombosis.

\section{Perspective}

The current article summarizes the 5-year follow-up data of the 30 first Perceval valves that were implanted. This experience is the first and longest with humans, with a truly sutureless valve, to evaluate implantation feasibility and valve safety. Results for up to 5 years of follow up confirmed the performance and safety of this device, even in a medium- to high-risk patient population. The valve did not reveal any dislodgement, structural valve deterioration, hemolysis, or thrombosis.

See Editorial Commentary page 88
The Perceval sutureless valve prosthesis (Sorin Group Italia S.r.l., Saluggia, Italy) has gained wide popularity both in minimal invasive and conventional aortic valve replacement

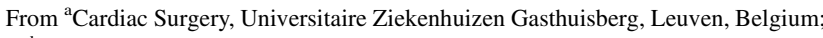
${ }^{\mathrm{b}}$ Cardiac Medico-Surgical Department, Institute Mutualiste Montsouris, Paris, France; and ${ }^{\mathrm{c} C a r d i o-T h o r a c i c ~ D e p a r t m e n t, ~ T r a n s p l a n t a t i o n ~ \& ~ V a s c u l a r ~ S u r g e r y, ~}$ Hannover Medical School, Hannover, Germany.

All funding for this trial was provided by Sorin Group Italia S.r.l., Saluggia, Italy.

Received for publication Oct 29, 2014; revisions received March 17, 2015; accepted for publication March 26, 2015; available ahead of print May 1, 2015.

Address for reprints: Bart Meuris, MD, PhD, Cardiac Surgery, U.Z. Gasthuisberg, Herestraat 49, 3000 Leuven, Belgium (E-mail: bart.meuris@uzleuven.be). 0022-5223/ $\$ 36.00$

Copyright (c) 2015 by The American Association for Thoracic Surgery http://dx.doi.org/10.1016/j.jtcvs.2015.03.040
}

to reduce aortic cross-clamp time and maximize effective valve orifice area by complete resection of the calcified aortic valve. Several studies demonstrated safety and efficacy of the valve prosthesis in both isolated and combined procedures. ${ }^{1-6}$ However, to date, only short-term results have been published, with outcomes of up to 12 months. In this paper, we present the midterm results (up to 5 years) for the first 30 patients included in this pilot trial (first use in humans) involving 3 centers.

\section{METHODS}

Approval for the study was granted by the institutional review boards of the university hospitals involved, and by the ethics committees of the hospitals; all patients provided written, informed consent. 


\section{Abbreviations and Acronyms \\ NYHA $=$ New York Heart Association \\ TAVI $=$ transapical or transfemoral aortic valve implantation}

\section{Study Design}

The pilot trial was designed as a prospective and nonrandomized study, on a maximum of 30 patients, and was conducted in 3 investigational centers (Hannover, Paris, and Leuven). The objective of the pilot trial was to assess the safety of aortic valve replacement with the sutureless Perceval valve in 30 symptomatic patients, aged $\geq 75$ years. The primary endpoint was the assessment of the safety of the prosthesis, in terms of mortality and morbidity at 30 days, correlated to prosthetic valve performance. Secondary endpoints were the evaluation of mortality and morbidity, the evaluation of the clinical status on the basis of the New York Heart Association (NYHA) functional classification, and the evaluation of the hemodynamic performance by echocardiographic examination at 1,3,6, and 12 months after implantation. All echocardiography data underwent echo-core lab review.

A minimum of 7 patients had to be enrolled at each investigational center. Inclusion criteria were being aged $>75$ years, and having aortic valve stenosis. The patients had to be eligible for standard surgical care. Further inclusion criteria were NYHA functional class III and/or IV, small $(<23$ $\mathrm{mm})$ and calcified aortic root or annulus. Exclusion criteria comprised aneurysmal dilation $(>4 \mathrm{~cm})$ of the ascending aorta, as well as an aortic annulus that was too large, as measured intraoperatively, after decalcification. During this experience with the first implantation in humans, only valve sizes $21 \mathrm{~mm}$ and $23 \mathrm{~mm}$ were available.

The enrollment was carried out in a sequential, prospective manner, such that all elderly patients with medium to high surgical risk were offered the possibility of participating in the assessment and were therefore evaluated according to the selection criteria defined in the protocol. Clinical and echocardiographic examinations were carried out at 1,3,6, and 12 months after implantation. At completion of the 12-month visits, follow-up of up to 5 years was implemented, to monitor the patients' clinical and hemodynamic status.

\section{Sutureless Valve}

The valve is a prosthesis consisting of a bovine pericardium valve fixed in a stent of superelastic alloy (equi-atomic alloy of nickel and titanium). The design features 2 ring segments, 1 on the proximal and 1 on the distal end, and connecting elements designed to support the valve and allow the prosthesis to anchor to the aortic root and the sinuses of Valsalva. Corresponding to each valve sinus, the inflow ring has 3 loops through which temporary guiding threads are passed, to aid the positioning of the prosthesis (Figure 1).

The superelastic alloy can undergo strong deformation and return to its original shape after the source of force is removed. Therefore, the stent can be compressed for the implantation and then released to reach its final diameter. Before implantation, the prosthesis is collapsed and loaded onto a holder. The valve is positioned and released in the aortic root, where the stent self-anchoring design allows stable seating of the device.

\section{Surgical Procedure}

Within the pilot trial, all patients underwent a standard median sternotomy. Under extracorporeal circulation and cross-clamping, a transverse aortotomy was performed $1 \mathrm{~cm}$ distal to the sinotubular junction (approximately $1 \mathrm{~cm}$ higher than a routine transverse aortotomy)

After the calcified native aortic valve was removed and the aortic annulus decalcified and measured, 3 guiding sutures were positioned 2 $\mathrm{mm}$ below the nadir of the native leaflet insertion line of each valve sinus. These sutures were passed through the corresponding eyelets in the prosthetic inflow ring, as a reference for alignment of the inflow section of the prosthesis with the insertion plane of the native leaflets.
The valve prosthesis was loaded onto the delivery device and inserted to the point where it was blocked by the temporary guiding sutures. The prosthetic valve was released in 2 phases: first, the inflow section of the valve, followed by the opening of the outflow part. To optimize the area of contact between the prosthesis and the aortic annulus, a postimplant dilatation was done with a specifically designed balloon catheter at pressures of $4 \mathrm{~atm}$ for 30 seconds, while warm water was applied to the valve. Once the prosthesis was completely deployed, the guiding sutures were removed.

After closure of the aortotomy in the usual fashion, and release of the aortic cross-clamp, the valve function was assessed by transesophageal echocardiography in all patients. After the procedure, the patients received anticoagulation treatment according to the standard protocol in use at each center for aortic bioprostheses.

\section{Reporting on Adverse Events and Statistical Analysis}

Adverse events were reported according to current guidelines. ${ }^{7}$ Analyses for descriptive statistics were done with SAS software, version 9.2 (SAS Institute, Cary, NC). All data are expressed as mean $\pm \mathrm{SD}$, or as median and quartiles, if not normally distributed. Kaplan-Meier analysis was performed for medium-term survival.

\section{RESULTS}

Between April 2007 and February 2008, a total of 30 patients underwent aortic valve replacement with the prosthesis. Characteristics of the patients and intraoperative data are given in Table 1. Three patients had undergone previous cardiac surgery.

Operative results and follow-up evaluation at 12 months have been reported previously. ${ }^{3}$ Follow-up evaluation was available on a yearly basis for up to 5 years. Cumulative follow-up time was 92.7 years. One patient died during hospital stay, from sudden cardiac arrest $(3.3 \%)$. Six patients in total died during the first postoperative years, between 45 and 1665 days after surgery, as reported previously, ${ }^{3}$ but only 1 of the deaths was valve related (endocarditis and sepsis on day 264). Overall 5-year survival was $71.3 \%$ (Figure 2).

Freedom from valve explant was $100 \%$ at 5-year followup evaluation. One patient had early mediastinal bleeding that led to tamponade and re-exploration on day 3, with a further uneventful course. Two late bleeding events occurred: 1 was gastrointestional bleeding that led to rehospitalization on day 36 , and 1 was retinal bleeding on day 350 without further treatment. One thromboembolic event was observed on day 6: limb ischemia, successfully treated with heparinization. Aside from the patient dying from severe endocarditis and sepsis, a second patient suffered from prosthesis endocarditis, leading to rehospitalization and complete resolution under antibiotic therapy on day 789 . One patient underwent pacemaker implantation early after surgery, owing to new-onset atrioventricular block.

Linearized rates (events per 100 patient-years) and actuarial probabilities of freedom from postoperative mortality and morbidity at 5 years were, respectively: late mortality, 6.5 (95\% confidence interval (CI) $1.5-11.5)$ and $71.3 \%$; bleeding, 2.2 (95\% CI 0.0-5.1) and 89.2\%; endocarditis, 


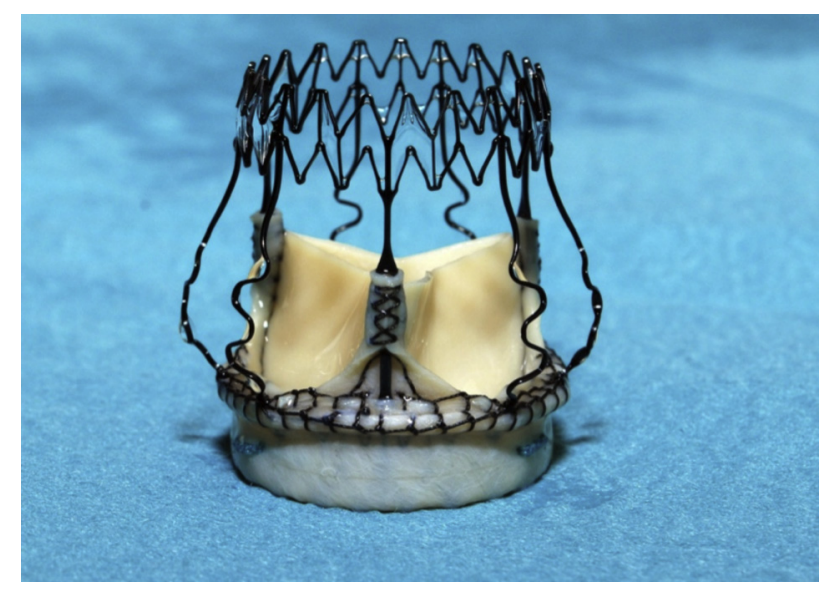

FIGURE 1. The Perceval sutureless aortic valve, as shown, is a bovine pericardial valve mounted into a superelastic alloy stent, with a doublering design (inflow ring at proximal side and outflow ring at distal side) and connecting elements designed to support the valve and allow the prosthesis to anchor to the aortic root and the sinuses of Valsalva.

2.2 (95\% CI 0.0-5.1) and 90.7\%; grade III atrioventricular block, 1.1 (95\% CI 0.0-3.2) and 91.0\%. No aortic regurgitation led to reoperation; 1 mild and 1 moderate perivalvular leak occurred at 12-month follow-up evaluation, and 1 mild perivalvular leak occurred at 5 years. No dislodgement of the valve, no structural valve deterioration, hemolysis, stroke, or valve thrombosis was observed within the 5year follow-up period.

Postoperative hemodynamics showed low mean and peak gradients, as well as an effective orifice area index that was revealed to be stable over time (Table 2). Functional results are given as postoperative NYHA class in Table 3. Taking into account that $93.3 \%$ of patients were in NYHA class III, and $6.7 \%$ in NYHA class IV preoperatively, most remained in NYHA class I and II during the follow-up period.
TABLE 1. Patient characteristics and intraoperative data

\begin{tabular}{lc}
\hline \multicolumn{1}{c}{ Characteristic } & $\begin{array}{c}\text { Perceval pilot } \\
\text { trial }(\mathbf{n = 3 0 )}\end{array}$ \\
\hline Gender, female/male & $22 / 8$ \\
Age at surgery (y) & $80.4 \pm 3.8(75-88)$ \\
Height $(\mathrm{cm})$ & $160.2 \pm 7.5(145-178)$ \\
Weight $(\mathrm{kg})$ & $70.5 \pm 14.9(47-110)$ \\
Body surface area $\left(\mathrm{m}^{2}\right)$ & $1.8 \pm 0.2(1.4-2.2)$ \\
Mean logistic euroSCORE & $13.18 \pm 7.28(5.48-39.07)$ \\
Concomitant coronary artery bypass grafting & 14 \\
Aortic valve stenosis & $23(76.7)$ \\
Combined aortic pathology & $7(23.3)$ \\
Implanted valve sizes (mm), & 11 \\
$\quad 21$ & 19 \\
$\quad 23$ & $63 \pm 11(45-85)$ \\
Left ventricular ejection fraction $(\%)$ & \\
Extracorporeal circulation time (min) & \\
$\quad$ Isolated aortic valve replacement & \\
$\quad$ Combined procedures & \\
Cross-clamp time (min) & \\
Isolated aortic valve replacement & $29.3 \pm 8.4 \pm 6.7(34-60)$ \\
Combined procedures & $45.4 \pm 15.4(21-79)$ \\
\hline
\end{tabular}

Values are given as mean $\pm \mathrm{SD}$ (range), or $\mathrm{n}(\%)$, unless otherwise indicated. euroSCORE, European System for Cardiac Operative Risk Evaluation.

\section{DISCUSSION}

For aortic valve stenosis, aortic valve replacement is still the treatment of choice. ${ }^{8}$ Transapical or transfemoral aortic valve implantation (TAVI) has been proposed as an alternative treatment in cases with high surgical risk. ${ }^{9}$ Because the TAVI approaches may occur with reduced valve orifice area and paravalvular leakage, owing to the fact that the calcified valve is left in place, alternative approaches, such as sutureless valves, have been reintroduced after the earliest attempts. In the late 1960s, the MagovernCromie prosthesis, a caged-ball mechanical prosthesis

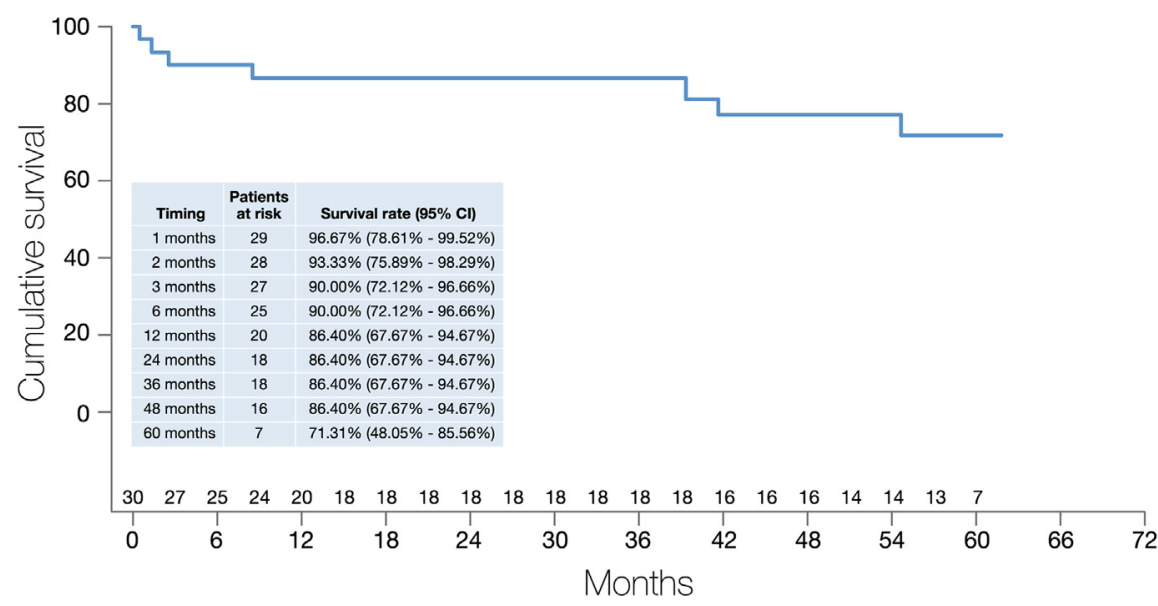

- Survival Probability

FIGURE 2. Overall survival is shown, of the first human study group $(n=30)$ up to 5 years, with a Kaplan-Meier curve. The numbers at risk are given at the bottom of the graph; $C I$, Confidence interval. 
TABLE 2. Hemodynamic performance (up to 5 years)

\begin{tabular}{|c|c|c|c|c|c|}
\hline & $12 \operatorname{mon}(n=23)$ & $2 y(n=18)$ & $3 y(n=12)$ & $4 y(n=11)$ & $5 y(n=10)$ \\
\hline Mean gradients (mm Hg) & $9.9 \pm 4.6$ & $8.0 \pm 4.1$ & $8.3 \pm 2.5$ & $7.6 \pm 3.6$ & $9.3 \pm 5.5$ \\
\hline Peak gradients (mm Hg) & $20.9 \pm 9.2$ & $16.6 \pm 7.2$ & $16.6 \pm 6.2$ & $17.5 \pm 7.8$ & $21.4 \pm 11.5$ \\
\hline Effective orifice area $\left(\mathrm{cm}^{2}\right)$ & $1.55 \pm 0.35$ & $1.51 \pm 0.26$ & $1.68 \pm 0.40$ & $1.68 \pm 0.43$ & $1.69 \pm 0.42$ \\
\hline Effective orifice area index $\left(\mathrm{cm}^{2} / \mathrm{m}^{2}\right)$ & $0.89 \pm 0.20$ & $0.77 \pm 0.22$ & $0.96 \pm 0.17$ & $0.96 \pm 0.27$ & $0.93 \pm 0.22$ \\
\hline
\end{tabular}

with anchoring spikes, was implanted, with acceptable results, even at long-term follow up. ${ }^{10,11}$

Various modern "sutureless" valve prototypes have been in introduced in recent years to reduce cross-clamp times: Most of the clinical experience to date has been with the ATS $3 \mathrm{f}$ Enable bioprosthesis ${ }^{12}$ (ATS, Minneapolis, Minn), the Edwards Intuity valve ${ }^{13}$ (Edwards LifeSciences, Irvine, Calif), and the Perceval sutureless aortic valve prosthesis ${ }^{3}$ (Sorin Group, Saluggia, Italy). The Perceval valve was introduced into clinical practice with a pilot trial (first use in humans, data within this article), followed by 2 prospective European trials (PIVOTAL [150 patients] and CAVALIER [658 patients]), both aiming at 5-year follow up with echocardiography core-lab reviewed data. At the latest 2014 European Association for Cardio-Thoracic Surgery meeting (abstract texts available through the organization website), data were shown from these trials, and the corresponding papers are in review. Data from a subgroup of patients with concomitant procedures have already been published. ${ }^{14}$ The first NorthAmerican experience occurred in Canada. ${ }^{15}$ In the United States, a prospective Investigational Device Exemption trial is running and has included 300 patients.

The surgical approach with full access to the aortic annulus allows for safe and complete decalcification of the annulus under direct vision to create a smooth annulus, thus preventing paravalvular leakages by proper fitting of the prosthesis into the annulus. The deployment system, together with dilatation of the valve, results in proper fixation without any valve dislodgement over time. The prosthesis can even be implanted in minimally invasive procedures, as demonstrated recently, ${ }^{16}$ and in redo cases, including calcified homografts. ${ }^{1,2}$

Our results confirm the safety and efficacy of the Perceval sutureless aortic valve, and for the first time, demonstrate excellent clinical and hemodynamic outcomes for up to 5 years, despite the advanced age of the enrolled population.

TABLE 3. Postoperative symptoms according to NYHA class

\begin{tabular}{lccccc}
\hline NYHA class & $\begin{array}{c}\text { Preoperative } \\
(\mathbf{n}=\mathbf{3 0})\end{array}$ & $\begin{array}{c}\mathbf{1 2} \text { mon } \\
(\mathbf{n}=\mathbf{2 3})\end{array}$ & $\begin{array}{c}\mathbf{2} \mathbf{y} \\
(\mathbf{n}=\mathbf{1 9})\end{array}$ & $\begin{array}{c}\mathbf{3} \mathbf{y} \\
(\mathbf{n}=\mathbf{1 4})\end{array}$ & $\begin{array}{c}\mathbf{4} \mathbf{y} \\
(\mathbf{n}=\mathbf{1 2})\end{array}$ \\
\hline I & $0(0.0)$ & 56.5 & 44.4 & 57.1 & 33.3 \\
II & $0(0.0)$ & 39.1 & 55.6 & 28.6 & 50.0 \\
III & $28(93.3)$ & 0 & 0 & 14.3 & 16.7 \\
IV & $2(6.7)$ & 0 & 0 & 0 & 0 \\
\hline
\end{tabular}

Values are \%, or n (\%). NYHA, New York Heart Association.
The valve implantation resulted in significant improvement of patients' symptoms, as well as reduction of transvalvular pressure gradients, which were shown to be stable over time. In addition, in patients requiring combined aortic valve replacement, along with concomitant procedures, sutureless valves may be advantageous, because the total cross-clamp times are significantly shorter.

The Perceval valve is currently commercially available throughout Europe. In addition, several countries (eg, Belgium, Germany, and Turkey) have approved reimbursement for this new prosthesis. The actual cost of the valve is higher than that for routine stented valves, but several groups have shown an overall reduction in hospital costs for patients who have used this device. ${ }^{17}$ The rapid deployment technique can lead to shorter operation and cardiopulmonary bypass times, and to faster postoperative recovery with shorter hospital stays.

The learning curve is short. Two proctored implantations are advised by the manufacturer. Paravalvular leakage can be avoided by proper decalcification of the annulus, proper placement technique, and thorough control of valve position visually and on transesophageal echocardiography during the weaning phase from cardiopulmonary bypass. Practically all centers that use the valve have seen a complete disappearance of paravalvular leaks in their growing experience with the device. The level of the aortotomy is slightly higher than the usual transverse incision for a routine stented valve, but the difference is subtle: An incision approximately $1 \mathrm{~cm}$ higher than the usual incision level is sufficient. An illustrative video segment, showing the entire implantation procedure, was previously published as supplemental material. ${ }^{6}$

Several centers have compared outcomes with sutureless valves to those with TAVI procedures (in moderate- to highrisk patients), revealing better results for sutureless valves regarding mortality, paravalvular leak rates, and vascular complications. ${ }^{18,19}$ In conclusion, this study reports the first and longest human experience with a truly sutureless valve, evaluating implant feasibility and valve safety. Results up to 5-year follow up confirmed the performance and safety of this device even in a medium- to high-risk patient population.

\section{Conflict of Interest Statement}

All funding for this trial was provided by Sorin. B. Meuris, F. Laborde, and M. Shrestha receive lecture fees from Sorin. 
All other authors have nothing to disclose with regard to commercial support.

\section{References}

1. Villa E, Messina A, Cirillo M, Brunelli F, Mhagna Z, Dalla Tomba M, et al. Perceval sutureless valve in freestyle root: new surgical valve-in-valve therapy. Ann Thorac Surg. 2013;96:e155-7.

2. Folliguet TA, Laborde F. Sutureless Perceval aortic valve replacement in aortic homograft. Ann Thorac Surg. 2013;96:1866-8.

3. Shrestha M, Folliguet T, Meuris B, Dibie A, Bara C, Herregods MC, et al. Sutureless Perceval S aortic valve replacement: a multicenter prospective pilot trial. $J$ Heart Valve Dis. 2009; 18:698-702.

4. Santarpino G, Pfeiffer S, Pollari F, Concistrè G, Vogt F, Fischlein T. Left ventricular mass regression after sutureless implantation of the Perceval S aortic valve bioprosthesis: preliminary results. Interact Cardiovasc Thorac Surg. 2014;18:38-42.

5. Folliguet TA, Laborde F, Zannis K, Ghorayeb G, Haverich A, Shrestha M. Sutureless Perceval aortic valve replacement: results of two European centers. Ann Thorac Surg. 2012;93:1483-8.

6. Flameng W, Herregods MC, Hermans H, Van der Mieren G, Vercalsteren M, Poortmans G, et al. Effect of sutureless implantation of the Perceval S aortic valve bioprosthesis on intraoperative and early postoperative outcomes. J Thorac Cardiovasc Surg. 2011;142:1453-7.

7. Akins CW, Miller DC, Turina MI, Kouchoukos NT, Blackstone EH, Grunkemeier GL, et al. Guidelines for reporting mortality and morbidity after cardiac valve interventions. J Thorac Cardiovasc Surg. 2008;135:732-8.

8. Beckmann A, Funkat AK, Lewandowski J, Frie M, Schiller W, Hekmat K, et al. Cardiac surgery in Germany during 2012: a report on behalf of the German Society for Thoracic and Cardiovascular Surgery. Thorac Cardiovasc Surg. 2014; 62:5-17.

9. Cribier A, Eltchaninoff H, Bash A, Borenstein N, Tron C, Bauer F, et al. Percutaneous transcatheter implantation of an aortic valve prosthesis for calcific aortic stenosis: first human case description. Circulation. 2002;106:3006-8.

10. Magovern GJ, Cromie HW. Sutureless prosthetic heart valves. J Thorac Cardiovasc Surg. 1963;46:726-36.
11. Totaro P, Nicolardi S, Zattera G, Viganò M. Old prosthesis with a modern concept: 41-year survival with a sutureless Magovern-Cromie aortic valve prosthesis. Eur J Cardiothorac Surg. 2011;39:789.

12. Martens S, Sadowski J, Eckstein FS, Bartus K, Kapelak B, Sievers HH, et al. Clinical experience with the ATS 3 f Enable Sutureless Bioprosthesis. Eur J Cardiothorac Surg. 2011;40:749-55.

13. Kocher AA, Laufer G, Haverich A, Shrestha M, Walther T, Misfeld M, et al. Oneyear outcomes of the Surgical Treatment of Aortic Stenosis With a Next Generation Surgical Aortic Valve (TRITON) trial: a prospective multicenter study of rapid-deployment aortic valve replacement with the EDWARDS INTUITY Valve System. J Thorac Cardiovasc Surg. 2013;145:110-5.

14. Shrestha M, Folliguet TA, Pfeiffer S, Meuris B, Carrel T, Bechtel M, et al. Aortic valve replacement and concomitant procedures with the Perceval valve: results of European trials. Ann Thorac Surg. 2014;98:1294-300.

15. Mazine A, Teoh K, Bouhout I, Bhatnagar G, Pelletier M, Voisine P, et al. Sutureless aortic valve replacement: a Canadian multicentre study. Can J Cardiol. 2015; 31:63-8.

16. Miceli A, Santarpino G, Pfeiffer S, Murzi M, Gilmanov D, Concistré G, et al. Minimally invasive aortic valve replacement with Perceval S sutureless valve: early outcomes and one-year survival from two European centers. J Thorac Cardiovasc Surg. 2014;148:2838-43.

17. Santarpino G, Pfeiffer S, Concistré G, Grossmann I, Hinzmann M, Fischlein T. The Perceval S aortic valve has the potential of shortening surgical time: Does it also result in improved outcome? Ann Thorac Surg. 2013;96:77-81.

18. Muneretto C, Bisleri G, Moggi A, Di Bacco L, Tespili M, Repossini A, et al. Treating the patients in the 'grey-zone' with aortic valve disease: a comparison among conventional surgery, sutureless valves and transcatheter aortic valve replacement. Interact Cardiovasc Thorac Surg. 2015;20:90-5.

19. Biancari F, Barbanti M, Santarpino G, Deste W, Tamburino C, Gulino S, et al. Immediate outcome after sutureless versus transcatheter aortic valve replacement. Heart Vessels. January 9, 2015 [Epub ahead of print].

Key Words: Aortic valve replacement, sutureless heart valve prosthesis

\section{EDITORIAL COMMENTARY}

\section{Encouraging durability results for sutureless aortic valve: The new gold standard for aortic valve replacement?}

Mattia Glauber, MD, and Antonio Miceli, MD, PhD

See related article on pages $84-8$.

From the Istituto Clinico Sant'Ambrogio, University and Research Hospitals, Gruppo Ospedaliero San Donato, Milano, Italy.

Disclosures: Mattia Glauber reports lecture fees, advisory boards, and proctorship from Sorin Group. The other author has nothing to disclose with regard to commercial support.

Received for publication April 17, 2015; accepted for publication April 17, 2015.

Address for reprints: Mattia Glauber, MD, Istituto Clinico Sant'Ambrogio, Gruppo Ospedaliero San Donato, University and Research Hospitals, Via Faravelli 16, 20100 Milano, Italy (E-mail: mattia.glauber@ftgm.it).

J Thorac Cardiovasc Surg 2015;150:88-90

$0022-5223 / \$ 36.00$

Copyright $\odot 2015$ by The American Association for Thoracic Surgery

http://dx.doi.org/10.1016/j.jtcvs.2015.04.047
Aortic valve replacement using stented valves is the conventional standard approach in the treatment of aortic valve disease. Despite the excellent long-term results with biological stented valves, sutureless technology recently has been developed as an alternative to stented valves in high-risk patients to simplify the surgical implantation and reduce the operative times. ${ }^{1-3}$

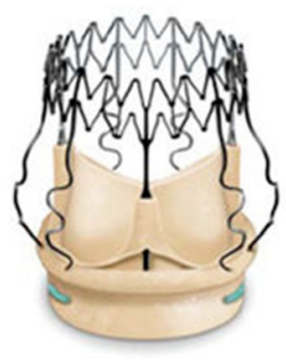

The Perceval S (Sorin Group, Milan, Italy) sutureless valve is new self-expanding prosthesis made of bovine pericardium mounted in a nitinol stent. Several studies 